\title{
Design of utility functions for game-based channel allocation in cognitive radio wireless sensor network
}

\author{
Prativa Rai ${ }^{1}$, Mrinal Kanti Ghose ${ }^{2}$, Hiren Kumar Deva Sarma ${ }^{3}$ \\ ${ }^{1,3}$ Sikkim Manipal Institute of Technology, Sikkim Manipal University, Sikkim, India \\ ${ }^{2}$ Department of Computer Engineering \& Applications, GLA University, Mathura (UP), India
}

\begin{tabular}{l} 
Article Info \\
\hline Article history: \\
Received Feb 1, 2021 \\
Revised Mar 15, 2021 \\
Accepted Mar 29, 2021 \\
\hline Keywords: \\
Cognitive radio \\
Cognitive radio wireless sensor \\
network \\
Game theory \\
Nash equilibrium \\
Utility function
\end{tabular}

\section{Article Info}

Article history

Received Feb 1, 2021

Revised Mar 15, 2021

\begin{abstract}
Cognitive radio enabled wireless sensor network is capable of reducing the spectrum scarcity problem of the wireless networks. Looking at the scarcity of available bandwidth, and the high growth in the number of communication devices in recent times, cognitive radio technology has proven to be a promising technology for the days to come. The application of game theory in cognitive radio networks has been visible in recent research works. However, only limited literature is available in which possibilities of applying the game-theory based approaches for the challenging task of channel assignment in cognitive radio wireless sensor are available in the literature. It is understood that the crux of the solution to the problem of scheming games for allocation of the channel is centered on the selection of the utility function in order to increase the efficiency of the channel allocation algorithm. Accordingly, the study regarding the influence of several utility functions on the performance of the corresponding channel allocation algorithm is important. Such a study enables designers to arrive at the optimal utility function to be used in game-theory based channel allocation algorithms, and the same is explored to the best extent, in this paper. The detailed procedure of allocating channels to all the contending nodes through game-based channel allocation has been discussed in this paper. Moreover, the performance of six different utility functions proposed which can be used for channel allocation using game theory has been evaluated for respective performances through MATLAB-based simulations.
\end{abstract}

This is an open access article under the CC BY-SA license.

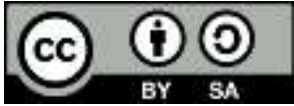

\section{Corresponding Author:}

Prativa Rai

Department of Computer Science \& Engineering

Sikkim Manipal Institute of Technology

Sikkim Manipal University, Sikkim, India

Email: prativa.r@smit.smu.edu.in

\section{INTRODUCTION}

Cognitive radio (CR) paradigm aims to solve the problem of spectrum scarcity by efficiently making use of under-utilized licensed spectrum [1], [2]. It allows the secondary users (SUs) to opportunistically use the spectrum holes formed by the licensed owners (PUs) [2]. The SUs must function without interfering with PUs. Wireless sensor networks (WSN) operate on unlicensed frequency bands and due to the tremendous increase in the usage of the unlicensed frequency band, they face a lot of performance degradation. Cognitive radio when applied to WSN can solve this problem. Therefore, several researchers have attempted to contribute towards the feasibility of applying cognitive radio to WSN, giving rise to the cognitive radio wireless sensor network (CRWSN) [3], [4]. 
The CRWSN comprises of CR enabled sensor nodes that possess the cognitive ability and reconfigurability properties [5]. The CR-enabled sensor nodes are the SUs and they can identify and use the unused licensed spectrum. However, these nodes have very limited energy, speed, and memory due to their size constraints [6]. Thus to embed CR functionality, the design of energy-efficient protocols that increase the network lifetime and performance of the overall network is needed.

In this paper, we focus on studying how underutilized frequency bands can be efficiently utilized by CR nodes in CRWSN by appropriate allocation of the same, i.e. the channel allocation problem. Several techniques can be applied while developing channel allocation protocols. In this work, a game theory (GT) based technique is adopted to allocate vacant channels efficiently. The SUs compete among themselves to get the vacant spectrum, hence this can be represented as a channel allocation game. Every game is defined by a set of players, strategy, and utility function. Every player has to select one move (strategy) from the strategy profile and based on the selected strategy, a player earns a payoff using the utility function. Hence, designing an appropriate utility function is very important as it leads to optimization in channel allocation.

Of late, the game theory principles have been gaining much popularity to resolute the various issues in several types of communication networks. The game theory appears applicable in CRWSN, as all the SUs try to latch on to the available slot in the spectrum given an opportunity, resulting in a definitive contention between the SUs for the unused spectrum. It is further challenging to amalgamate the concepts of CR and game theory in a single platform. Since the concept of CRWSN is itself relatively new and is in a natal phase of development at the moment, very limited works in this area have been reported so far and are available in the literature.

In [7], the authors have investigated the prevailing game-based methodologies for WSN. In the same work, a classification of future research possibilities has also been presented. Several policies also have been presented in [8]-[10] for game-based channel allocation in cognitive radio networks to enhance the overall throughput of the network. A game-based resource allocation with energy harvesting for CRWSN has been proposed by $\mathrm{Xu}$, et al. [11] wherein the SUs have been augmented with energy harvesting circuitry. A framework for CRWSN using game theory has been suggested in [12]. In game-based algorithms, the formulation of the utility function plays an important role. The better is the utility function the better is the proposed algorithm [13]. From the available literature [14]-[20], it has been observed that most of the researchers have considered (i) bit error rate (BER) (ii) signal-to-interference-plus-noise ratio (SINR), (iii) energy consumption (iv) throughput, and (v) quality of service network metrics to formulate the utility function.

The rest of the paper is organized as follows: The proposed work has been provided in Section 2 where the system model has been outlined. The research method has been presented in Section 3 where the utility functions using different network metrics have been derived for channel allocation and it has been explained with elaborate examples. In Section 4, simulation results are analyzed and we conclude the findings of this paper in Section 5.

\section{PROPOSED WORK}

In this paper, an attempt has been made to explore various utility functions based on different network metrics. Each utility function has been examined thoroughly with suitable examples, and the impacts of those parameters resulting in nash equilibrium (NE) are analyzed. A preliminary version of this paper was presented in [21].

The main contributions made in this paper are stated:

a) Six utility functions for game-based channel allocation have been analyzed through numerical examples.

b) A generalized algorithm for channel allocation in CRWSN making use of game theory is outlined.

c) Performance evaluation of the six utility functions is carried out through MATLAB-based simulations.

d) The best utility function is identified, based on experiments.

\subsection{System model}

A CRWSN with $N$ cognitive radio (CR) nodes or the secondary users (SU) uniformly deployed in a $\mathrm{D} \times \mathrm{D}$ area has been considered for the proposed study. The SUs can exchange data packets with the neighboring nodes within their range of transmission. Assuming there are $C(<N)$ channels owned by as many primary users (PU). The number of users is more compared to the licensed channel which leads to competition among the SUs to access the licensed band. For simplicity, we assume that PUs are inactive in the sense that PUs are not transmitting while SUs are contending for the channels. However, the PU can transmit, if it chooses to, resulting in PU reoccupying its channel. When two or more SUs use the same channel at the same time then interference occurs. Most of the contemporary research works reveal that the SINR value is most suitable to check the interference level. For measuring the SINR, a bi-directional 
interference denoted by $I_{\text {to }}$ and $I_{\text {from }}$ has been considered, wherein $I_{\text {to }}$ is the interference a node produces for other nodes and $I_{\text {from }}$ is the interference a node suffers from other nodes. Following [15], [22], [23] the SINR between a transmitting node $t$ and a receiving node $r$ can then be given by (1).

$$
S I N R_{t r}=\frac{p_{t} h_{t r}}{\sum_{r=1 \neq t}^{N} p_{r} h_{r t}+n}
$$

where, $p_{t}$ is the transmitting power of node $t, h_{t r}$ is the path loss between transmitting node $t$ and a receiving node $r$. Considering free space propagation, the path loss is expressed as $1 / d_{t r}^{2}$ where $d_{t r}$ is the distance between a transmitting node $t$ and a receiving node $r$, and $n$ is the noise power. When the CR nodes use the same channel, then they create interference to the nodes falling within its transmission range. Based on (1), the values of $I_{\text {to }}$ and $I_{\text {from }}$ for node $i$ can be obtained using the (2) and (3).

$$
\begin{aligned}
& I_{\text {to }}=\sum_{j=1 \neq i, c=c}^{N} p_{i} h_{i j} \\
& I_{\text {from }}=\sum_{j=1 \neq i, c=c}^{N} p_{j} h_{j i}
\end{aligned}
$$

The feasibility of selecting a channel is indicated by the interference level and is inversely proportional to each other. Accordingly, a high utility is expected for low channel interference and viceversa. The worst-case scenario being all nodes competing for a single vacant channel where the highest interference is expected. Thus, it is necessary to balance the spectrum usage pattern efficiently.

\section{RESEARCH METHOD}

In view of the above justifications, it is proposed to derive the utility functions using interference along with other network metrics as an input and to access those functions in the succeeding section.

\subsection{Utility functions for channel allocation game}

A game-based channel allocation can be formulated as $G=\langle P, S, u\rangle$ where $P$ represents the player set, $S$ denotes the action space or strategy profile, and $u$ represents the utility function of the game.

a) $\quad$ Player $\operatorname{Set}(P)$ denoted as $\mathrm{P}=\{1,2, \cdots, \mathrm{N}\}$ indicates the set players in the game which in this work are the N CR nodes.

b) Action Space $(S)$ denoted by $S=\left\{s_{1}, s_{2}, \cdots, s_{N}\right\}$ represents a set of strategies/actions a player can select. The strategy selected by a node $i$ where $i \in P$, is denoted by $s_{i}$ and the strategy selected by other nodes except $i$ is denoted by $s_{-i}$.

c) Utility function $(u)$ determines the objective of the game in terms of payoff. A node earns a certain payoff when the game is played with a strategy derived from $S$. The utility function of a player $i$ is indicated as $u_{i}\left(s_{i}, s_{-i}\right)$

Each player targets to achieve maximum payoff during the game. However, eventually, a player reaches a point in the game where it cannot further attain a greater payoff than the current payoff maximum, even by altering its strategy. This point in the game is known as nash equilibrium (NE). For a strategy profile $S=\left\{s_{1}, s_{2}, \cdots, s_{N}\right\}, \mathrm{NE}$ is defined as;

$$
u_{i}\left(s_{i}^{*}, s_{-i}^{*}\right) \geq u_{i}\left(s_{i}^{\prime}, s_{-i}^{*}\right) \quad \forall i \in N, \forall s^{\prime} \in S_{i}
$$

where $s_{i}^{*}$ denotes player $i^{\prime} s$ best response strategy and $s_{-i}^{*}$ denotes the best response of the remaining players excluding $i$ [24]. It is appropriate to mention here that the terms 'node', 'user', and 'player' can be used interchangeably.

In the proposed game, the action space $S$ will comprise the different grouping of available unused channels concerning players in the game. For example, if $C=L$, then the action space will be $L^{N}$. Based on the objective of the algorithm, the utility function can be derived by incorporating different network metrics. Some of the important utility functions have been deduced below by incorporating different network metrics under several network situations. The preliminary work on the same has been presented in [21] and the network scenario remains the same as shown in Figure 1. The nodes 1, 2, and 3 are communicating with 4 and the game parameters can be obtained as follows:

$$
P=\{1,2,3\}
$$

$S=2^{3}=\{C 1, C 1, C 1 ; C 1, C 1, C 2 ; C 1, C 2, C 1 ; C 1, C 2, C 2 ; C 2, C 1, C 1 ; C 2, C 1, C 2 ; C 2, C 2, C 1 ; C 2, C 2, C 2\}$. 
Here, Node 4 does not play the game, therefore, does not contend for the channel. The strategy $S=$ $\{C 1, C 1, C 1\}$ shows that all the players select $C 1$ channel.

In [21], three utility function indicated by Case I to Case III were derived which is listed below:

Case I: Considering only SINR as the network metric

In the first case, only the SINR metric is used to derive the utility function. Consider a node $i$ selects a strategy of using channel $c$ then the utility function can be expressed by using (1) as:

$$
u_{i c}\left(s_{i}, s_{-i}\right)=\frac{p_{i} h_{i j}}{\sum_{j=1 \neq i, s_{i}=s_{j}}^{N} p_{j} h_{j i}+n}
$$

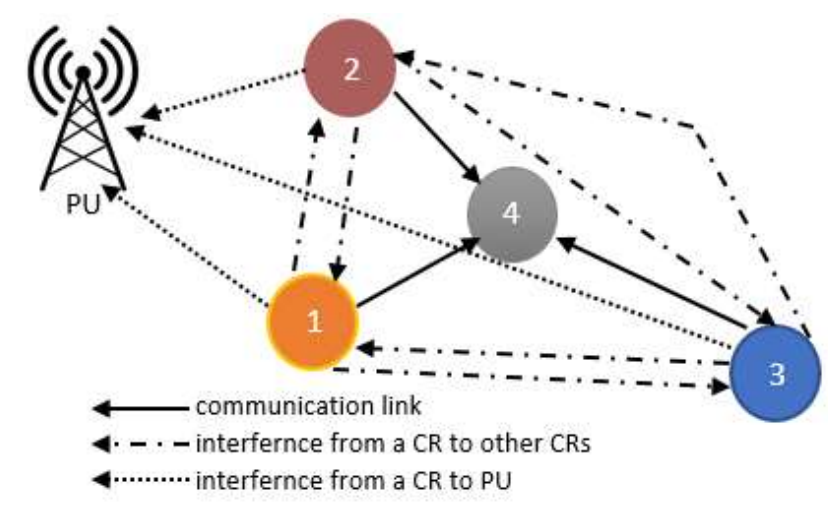

Figure 1. CRWSN scenario

\section{Case II: Considering $I_{\text {to }}$ and $I_{\text {from }}$ as network metrics}

Every node aims to lower the interference caused to other nodes and interference suffered from other nodes and increase its Rx power, thus it is worth considering both $I_{\text {to }}$ and $I_{\text {from }}$ in developing the utility function as shown in (6).

$$
u_{i}\left(s_{i}, s_{-i}\right)=-\alpha I_{\text {from }}-\beta I_{t o}+p_{i}-n
$$

Where $\alpha, \beta$ are some constants such that $\alpha+\beta \leq 1$.

\section{Case III: Considering $I_{\text {to }}, I_{\text {from }}$ and remaining energy as network metric}

The low-energy nodes expire early in comparison to nodes with higher energy. Besides, the amount of interference effect from low energy nodes is high [17]. Therefore, the other competing nodes will avoid selecting the channel used by nodes having low energy. As a result, the channel allocated to low energy nodes is not shared thereby allowing such nodes to complete their transmission before they die. Thus, the remaining energy ( $r e$ ) of nodes is considered while designing the utility function. The terms $I_{\text {from }}$ and $I_{t o}$ are reformulated by considering $r e$ as given in (7) and (8).

$$
\begin{aligned}
& I_{\text {from }}=\sum_{j=1 \neq i, s_{i}=s_{j}}^{N} \frac{p_{j h_{j i}}}{r e_{j}} \\
& I_{\text {to }}=\sum_{j=1 \neq i, s_{i}=s_{j}}^{N} \frac{p_{i h_{i j}}}{r e_{i}}
\end{aligned}
$$

It is seen from (7) and (8), that low energy node is seen to produce high interference in comparison with high-energy nodes. By replacing the values of $I_{\text {from }}$ and $I_{\text {to }}$ as calculated using (7) and (8) in (6), the values of payoffs under different scenarios can be obtained. In this case, the following two scenarios have been studied.

Case III-A: All nodes have equal remaining energy.

Case III-B: All nodes have varying remaining energy.

From the results obtained for Case I, II, and III-A \&B in [21], it is observed that for Case I, II, and III-A the Nash equilibrium were achieved while playing strategy $\{\mathrm{C} 1, \mathrm{C} 2, \mathrm{C} 2\}$ and $\{\mathrm{C} 2, \mathrm{C} 1, \mathrm{C} 1\}$ whereas for Case III-B the strategy $\{\mathrm{C} 1, \mathrm{C} 1, \mathrm{C} 2\}$ and $\{\mathrm{C} 2, \mathrm{C} 2, \mathrm{C} 1\}$ lead to Nash equilibrium. Further, it may be concluded 
that $\mathrm{Tx}$ nodes nearby to their Rx node, seemingly get a single channel whereas others share a common channel. However, in Case III-B, it has been observed that closer nodes are allocated the same channel, regardless of their distance from the Rx as exhibited in earlier cases. Thus, it can be seen that the nodes avoid sharing the channel with low energy nodes. As a result, the low-energy nodes get full access to the channel.

\section{Case IV: Considering $I_{\text {to }}, I_{\text {from }}$, remaining energy and cost as network metric}

In this case, we have introduced a cost function as an added parameter in designing the utility function. The cost function $C_{i c}$ denotes the cost of using a channel $c$ by node $i$ where $c \in[1, C]$. The cost function has been derived considering the factors mentioned below:

i) Amount of interference a node/player creates over a channel: A node causing higher interference $\left(I_{t o}\right)$ over a channel will be penalized with higher cost whereas a node causing lower interference is charged with lower cost.

ii) Amount of interference suffered from other nodes $\left(I_{\text {from }}\right)$ : All the nodes try to use the vacant channel or the channel having a lower interference level. Therefore, if $I_{\text {from }}$ is high then it indicates that the channel was used by many other nodes so a node will avoid selecting such a channel. So, the cost of such a channel should be lowered.

iii) The number of available channels $(C)$ : If the number of available channels is more then the strategy profile increases. So, each node has a larger pool of channels to select from thereby the cost per channel decreases.

Hence, we formulate the cost function using the above factors for node $i$ using channel $c$ as shown in (9).

$$
C_{i c}=I_{t o} / I_{\text {from }} \times C
$$

Using the above cost function, the utility function for node $i$ selecting strategy $s_{i}$ using channel $c$ derived in (6) using (7) and (8) can be reformulated as:

$$
u_{i c}\left(s_{i}, s_{-i}\right)=-\alpha I_{\text {from }}-\beta I_{t o}+p_{i}-n-C_{i c}
$$

Considering the same scenarios used in the above cases and keeping the remaining energy of all nodes the same, the payoff of each node is calculated and presented in Table 1. The resultant payoff matrix has been shown in Table 2. Subsequently, the energy levels can be varied in line with Case III-B.

Table 1. Payoff table for case IV

\begin{tabular}{ccccccc}
\hline \multicolumn{1}{c}{ Strategy } & \multicolumn{5}{c}{ Payoff } \\
Node 1 & Node 2 & Node 3 & Node 1 & Node 2 & Node 3 & NE Achieved \\
\hline C1 & C1 & C1 & -4.29052 & -0.47685 & -0.3132 & No \\
C1 & C1 & C2 & $\mathbf{- 3 . 9 3 7 1 2}$ & $\mathbf{- 0 . 0 4 1 3 3}$ & $\mathbf{0 . 9}$ & Yes \\
C1 & C2 & C1 & -4.48207 & 0.9 & 0.049123 & No \\
C1 & C2 & C2 & 0.9 & -1.38602 & -0.94755 & No \\
C2 & C1 & C1 & 0.9 & -1.38602 & -0.94755 & No \\
C2 & C1 & C2 & -4.48207 & 0.9 & 0.049123 & No \\
C2 & C2 & C1 & $\mathbf{- 3 . 9 3 7 1 2}$ & $\mathbf{- 0 . 0 4 1 3 3}$ & $\mathbf{0 . 9}$ & Yes \\
C2 & C2 & C2 & -4.29052 & -0.47685 & -0.3132 & No \\
\hline
\end{tabular}

Table 2. Payoff matrix for case IV

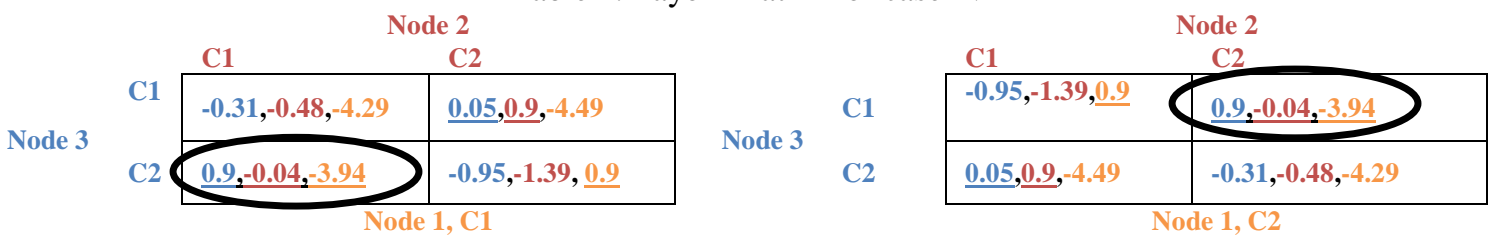

The best strategy of each player is considered and it is perceived that there are two NE i.e $\{\mathrm{C} 1, \mathrm{C} 1$, $\mathrm{C} 2\}$ and $\{\mathrm{C} 2, \mathrm{C} 2, \mathrm{C} 1\}$. It indicates that the nearby nodes share a common channel. Using cost factor, it is observed that the utility values of nodes closer to each other reduce since they produce more interference as compared to distant nodes.

Case V: Using $I_{\text {to }}, I_{\text {from }}$, remaining energy, cost, and PU's detection

The utility function derived in all the above cases (I-IV) assumes that PUs are inactive (not present) but it may not happen always. There will be some percentage of error in detecting the presence of PUs raising 
a false alarm [25]. If PU is present in the channel then interference from PU $\left(I_{\text {from }}^{P U}\right)$ and interference to PU $\left(I_{t o}^{P U}\right)$ needs to be considered as well. Therefore, the utility function for node $i$ selecting strategy $s_{i}$ can be redesigned as:

$$
u_{i c}\left(s_{i}, s_{-i}\right)=-(1-\varphi)\left[\alpha I_{\text {from }}+\beta I_{t o}\right]-\varphi\left[I_{\text {from }}^{c}+I_{\text {to }}^{c}\right]+p_{i}-n-C_{i c}
$$

Where $\varphi$ the probability of miss detection of PU and interference of PU is is computed as:

$$
\begin{aligned}
& I_{\text {from }}^{c}=\sum_{m=1, s_{m}=c}^{N} p_{m} h_{m P} \\
& I_{\text {to }}^{c}=\sum_{m=1, s_{m}=c}^{N} p_{P} h_{P m}
\end{aligned}
$$

The rest parameters remain the same as used in earlier cases.

Here, it has been assumed that the probability of miss detection is very small $(\varphi=0.2)$ and the transmit power of PUs is 10 watts. Under the same network scenario and assumption used in Case IV, the payoff values of each node under different strategies are calculated using (11) and shown in Table 3.

Table 4 shows the payoff matrix achieved from the payoff values obtained. It is observed that there $t$ two NE exist i.e $\{\mathrm{C} 1, \mathrm{C} 1, \mathrm{C} 2\}$ and $\{\mathrm{C} 2, \mathrm{C} 2, \mathrm{C} 1\}$. The nodes in close proximity share the same channel. Also, by varying the value of $\varphi$ it is seen that the best strategy does not change and results in the same two NE

\begin{tabular}{|c|c|c|c|c|c|c|}
\hline \multirow[b]{2}{*}{ Node 1} & \multicolumn{2}{|l|}{ Strategy } & \multicolumn{3}{|c|}{ Payoff } & \multirow{2}{*}{ NE Achieved } \\
\hline & Node 2 & Node 3 & Node 1 & Node 2 & Node 3 & \\
\hline $\mathrm{C} 1$ & $\mathrm{C} 1$ & $\mathrm{C} 1$ & -4.44908 & -0.6275 & -0.46324 & No \\
\hline C1 & C1 & $\mathrm{C} 2$ & -4.0648 & -0.16108 & 0.779452 & Yes \\
\hline $\mathrm{C} 1$ & $\mathrm{C} 2$ & $\mathrm{C} 1$ & -4.58171 & 0.812871 & -0.04199 & No \\
\hline $\mathrm{C} 1$ & $\mathrm{C} 2$ & $\mathrm{C} 2$ & 0.83125 & -1.46797 & -1.14945 & No \\
\hline $\mathrm{C} 2$ & $\mathrm{C} 1$ & $\mathrm{C} 1$ & 0.799229 & -1.44153 & -1.00246 & No \\
\hline $\mathrm{C} 2$ & $\mathrm{C} 1$ & $\mathrm{C} 2$ & -4.5416 & 0.718762 & -0.00188 & No \\
\hline $\mathrm{C} 2$ & $\mathrm{C} 2$ & C1 & -3.96741 & -0.06369 & 0.752182 & Yes \\
\hline $\mathrm{C} 2$ & $\mathrm{C} 2$ & $\mathrm{C} 2$ & -4.54793 & -0.72634 & -0.56208 & No \\
\hline
\end{tabular}
states. It indicates that the distance of PUs does not affect the performance of the network.

Table 3. Payoff table for case V

Table 4. Payoff matrix for case $\mathrm{V}$

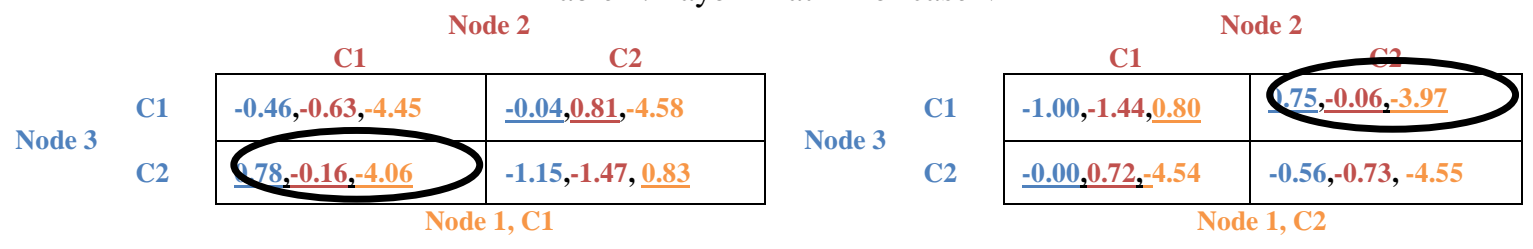

\section{Case VI: Using Throughput}

The connection established between the Tx node and the Rx node is called a link. The quality of a link also plays an important role in deciding the strategy by the nodes. To assess the quality of the link between two nodes, the throughput of a link has been considered. The throughput of a link $k$ based on shannon capacity with additive white gaussian noise [26] is expressed as;

$$
T_{k}=B * \log _{2}\left(1+S N R_{k}\right)
$$

Where B is the channel bandwidth and SNR is the signal-to-noise ratio. Bhattarai et al. [10] have normalized throughput with respect to bandwidth and is expressed as;

$$
T_{k}=\log _{2}\left(1+S I N R_{k}\right)
$$

Using only the throughput of a link, the utility function of a node $i$ using channel $c$ and node $i$ is a part of link $k$ is expressed as; 


$$
u_{i c}\left(s_{i}, s_{-i}\right)=T_{k}
$$

Considering the utility function, the payoff values and its corresponding payoff matrix is presented in Table 5 and Table 6 respectively.

Table 5. Payoff table for case VI

\begin{tabular}{ccccccc}
\hline \multicolumn{3}{c}{ Strategy } & \multicolumn{5}{c}{ Payoff } \\
Node 1 & Node 2 & Node 3 & Node 1 & Node 2 & Node 3 & NE Achieved \\
\hline C1 & C1 & C1 & 1.10112 & 0.36822 & 0.32540 & No \\
C1 & C1 & C2 & 1.59471 & 0.47846 & 2.62792 & No \\
C1 & C2 & C1 & 1.68532 & 2.76136 & 0.43563 & No \\
C1 & C2 & C2 & $\mathbf{3 . 8 7 7 6 1}$ & $\mathbf{0 . 9 5 2 4 3}$ & $\mathbf{0 . 8 1 8 9 9}$ & Yes \\
C2 & C1 & C1 & $\mathbf{3 . 8 7 7 6 1}$ & $\mathbf{0 . 9 5 2 4 3}$ & $\mathbf{0 . 8 1 8 9 9}$ & Yes \\
C2 & C1 & C2 & 1.68532 & 2.76136 & 0.43563 & No \\
C2 & C2 & C1 & 1.59471 & 0.47846 & 2.62792 & No \\
C2 & C2 & C2 & 1.10112 & 0.36822 & 0.32540 & No \\
\hline
\end{tabular}

From the payoff matrix, two NE strategy i.e. $\{\mathrm{C} 1, \mathrm{C} 2, \mathrm{C} 2\}$ and $\{\mathrm{C} 2, \mathrm{C} 1, \mathrm{C} 1\}$ are obtained. It indicates that shorter links share the same channel compared to longer links.

Table 6. Payoff matrix for case VI
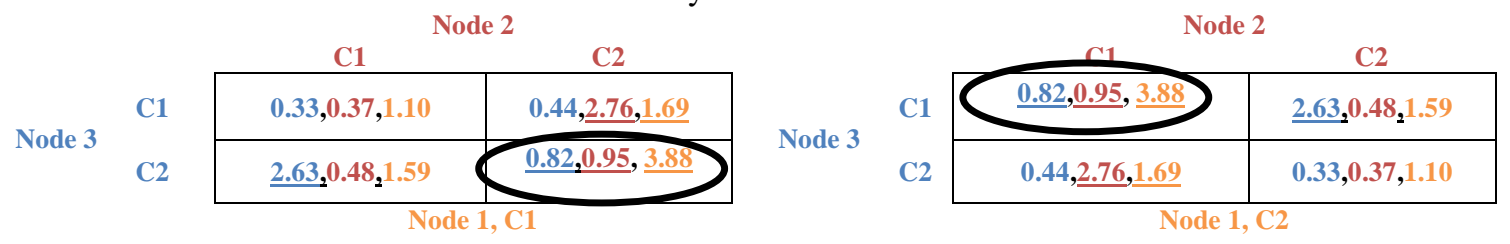

\subsection{A generalized strategy to allocate channel in CRWSN on the basis of game theory}

As deliberated in the above Cases I to VI, the best-response strategy converges to Nash equilibrium. Therefore, a Game Theory-based generalized algorithm for channel allocation in CRWSN, as shown in Figure 2 can be obtained. The SUs play the game in a round-robin fashion. So, SU1 monitors the game and selects a strategy from the action space.

Using the utility function, it computes the new payoff value. It changes its strategy only if it gains a higher payoff compared to the previous payoff. After SU1 makes its decision then SU2 scans the game and makes its choice based on the best response. This process is repeated for all SUs. The algorithm continues for a specific number of iterations or until it converges to Nash equilibrium.

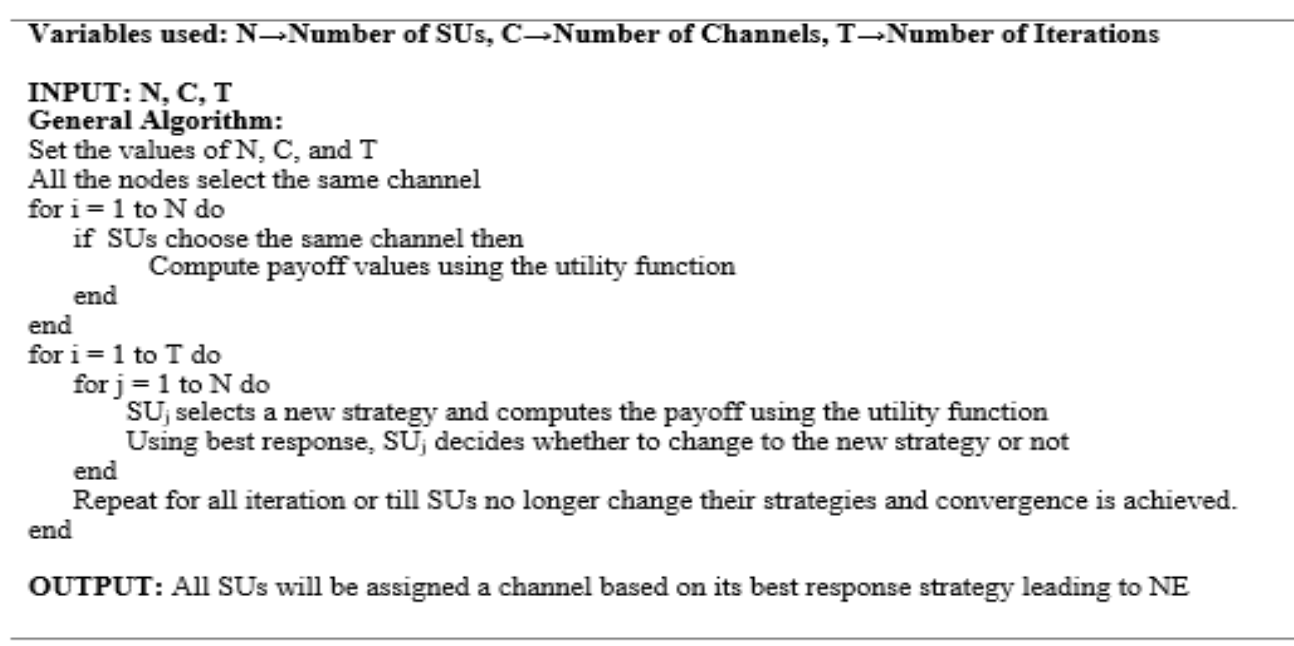

Figure 2. Game-based channel allocation algorithm (a generalized version) 


\section{SIMULATION RESULTS}

The simulations using MATLAB (R2015a) are carried out for different cases as mentioned in Section 3 on the system model as described in Section 2.1 and the several simulation results are presented below. For the present study, it is assumed that three players are contending for accessing two licensed channels. So a game has the following attributes $P=\{N 1, N 2, N 3\}$ and $C=\{C 1, C 2\}$, for all cases mentioned in Section 3. To be more specific, there are 3 SUs and 2 PUs. It is further considered that the communication range is $50 \mathrm{~m}$, and the transmitting and receiving power for all the nodes are constant the value of which is assigned as 1 .

For the above-assigned values, the channel usage patterns are shown in Figure 3 to Figure 10. From Figure 3, it can be seen that before channel allocation, the same channel $C 1$ is used by all the nodes for communication with N4 irrespective of whichever algorithm is used. Figure 4 to Figure 10 show the channel allocation for three nodes at Nash equilibrium as followed in Cases I, II, III-A, III-B, IV, V, and VI respectively. Figure 4 of Case I, Figure 5 of Case II, Figure 6 of Case III-A, and Figure 10 of Case VI show that the node near its destination node, is assigned a single channel and other nodes share a common channel. However, Figure 7 for Case III B, Figure 8 of Case IV, and Figure 9 of Case V for varying energy levels reveal that the low energy node is assigned an exclusive channel irrespective of its distance from its destination node. Figure 11 depicts the level of interference in each node before the allocation of the channel, while Figure 12 shows the corresponding values post channel allocation for all six cases. A drastic drop in interference levels post channel allocation can be ascertained from Figures 11 and 12.

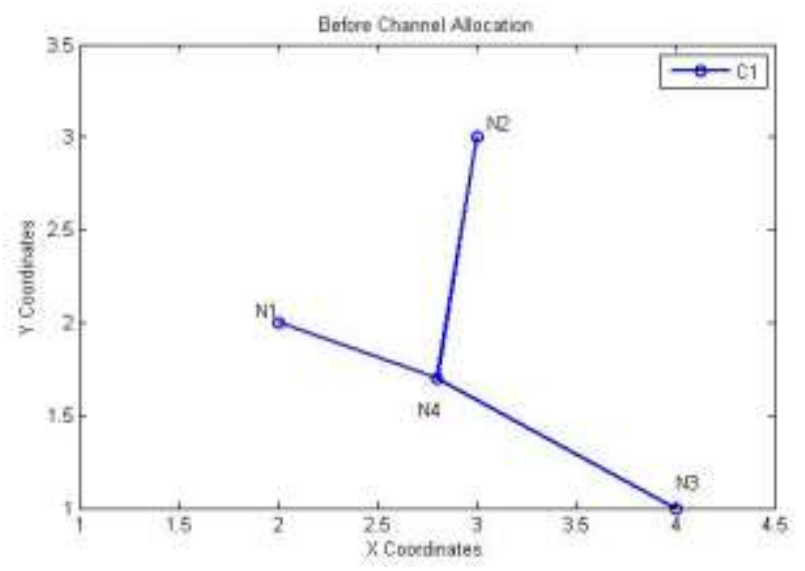

Figure 3. Before channel allocation

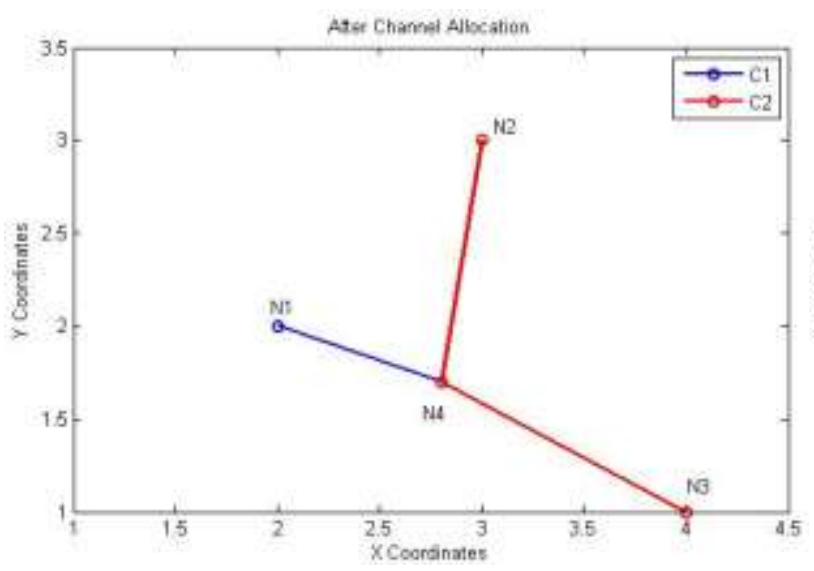

Figure 5. After channel allocation (Case II)

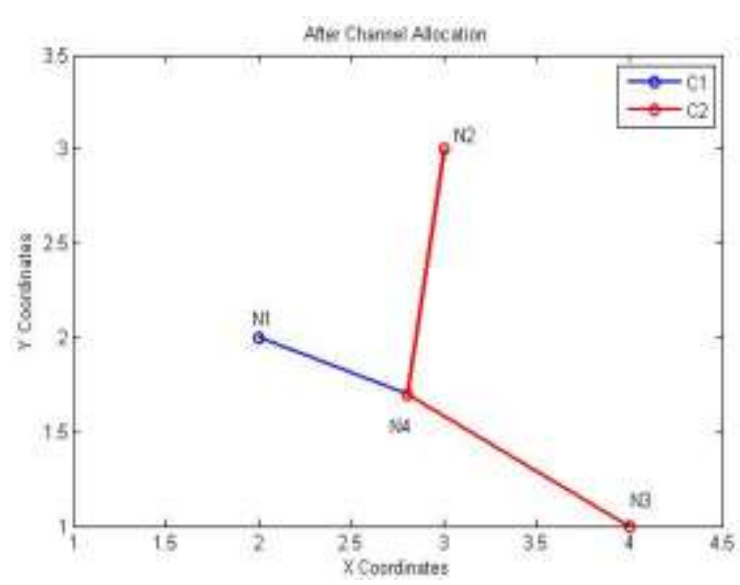

Figure 4. After channel allocation (Case I)

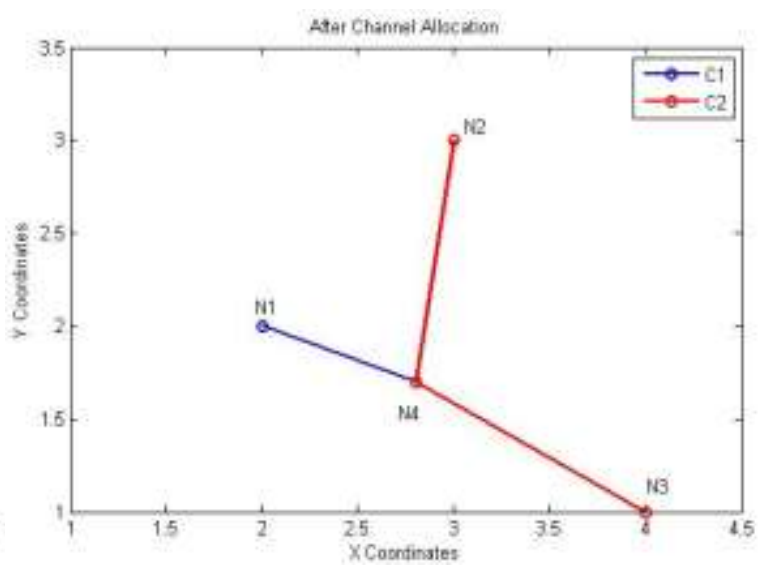

Figure 6. After channel allocation (Case III-A) 


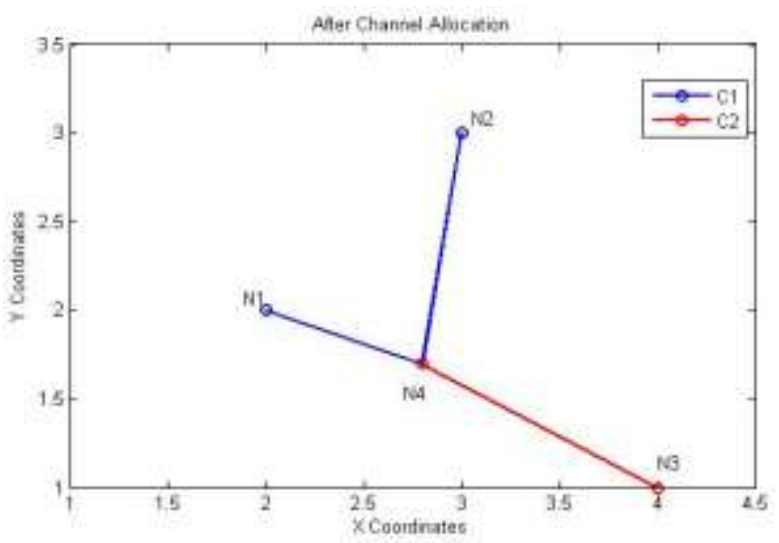

Figure 7. After channel allocation (Case III - B)

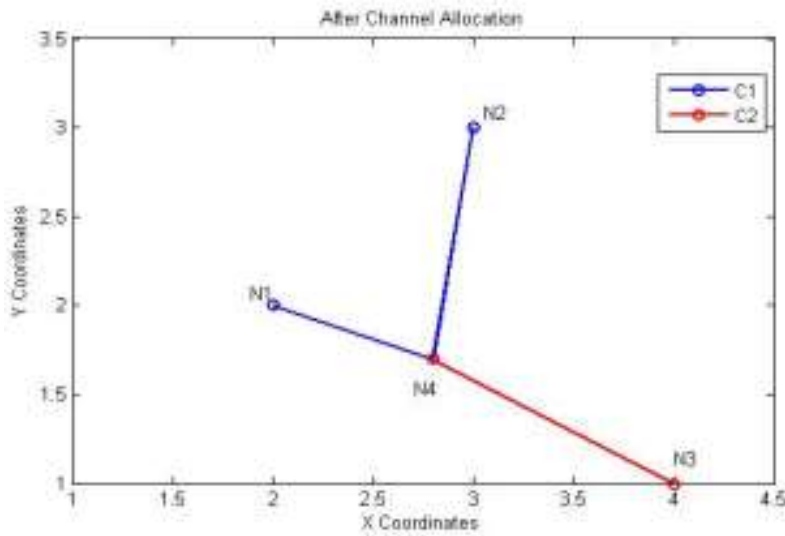

Figure 9. After channel allocation (Case V)

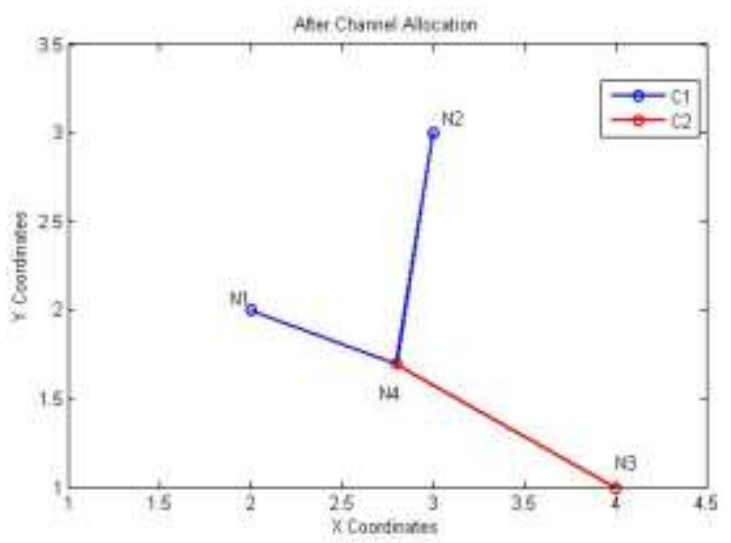

Figure 8. After channel allocation (Case IV)

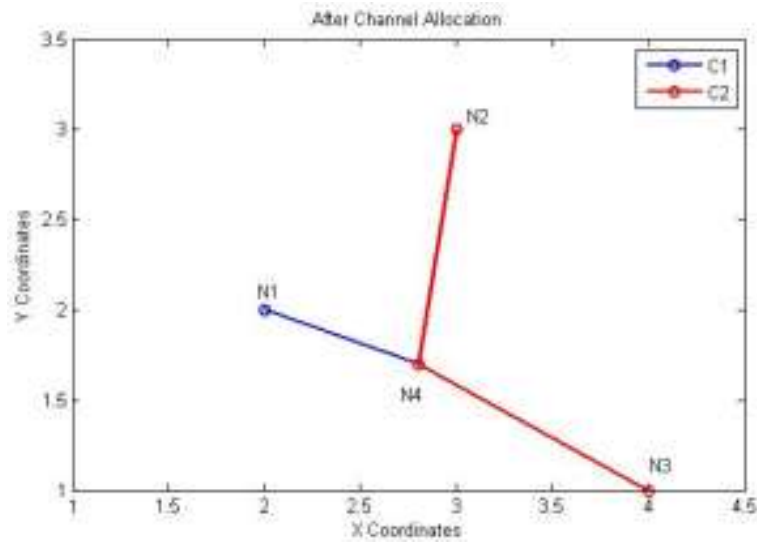

Figure 10. After channel allocation (Case VI)

From the experiments, the nethermost interference level is obtained for Cases IV and V as shown in Figure 12. This is due to the incorporation of the cost factor in the design of the utility function in Case IV and the probability of PU detection in Case V. The process, thus emerges as a superior channel allocation mechanism in the game. The second best channel allocation policy is Case III where the remaining energy of nodes is incorporated. Then using SINR and Throughput i.e. Case I and Case VI respectively, give the thirdbest channel allocation policy. However, Case II exhibits the highest interference among all the cases. In Case II, $I_{\text {to }}$ and $I_{\text {from }}$ metrics have been used in the utility function. Therefore, Case II is the least preferred option where the lowest interference level is preferred for the channel allocation policy.

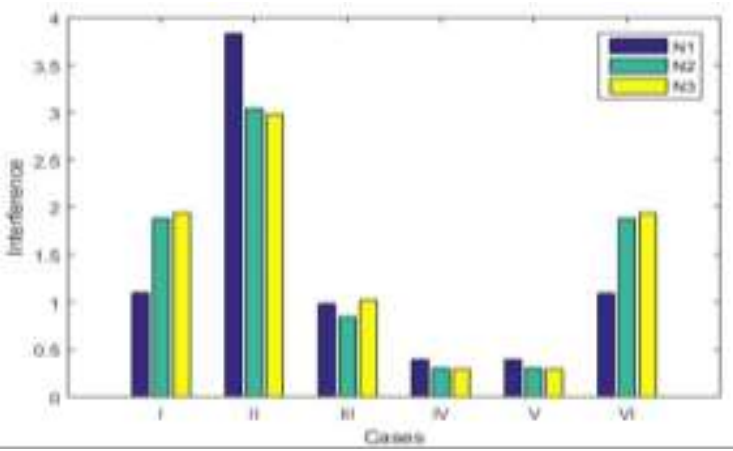

Figure 11. Interference level before channel allocation

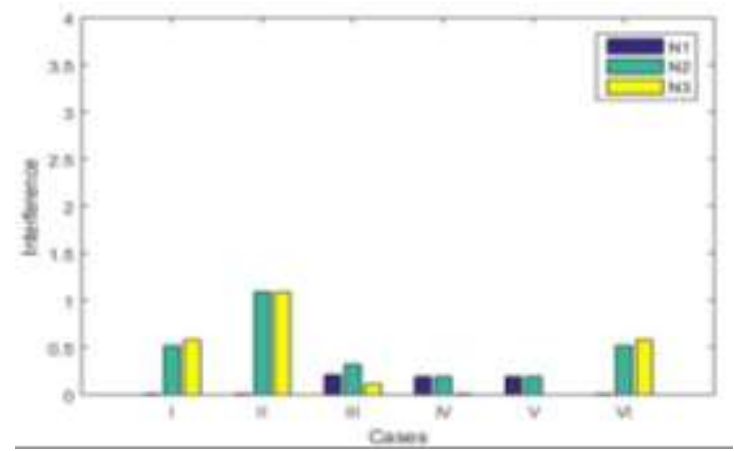

Figure 12. Interference level after channel allocation 


\section{CONCLUSION}

Applications of Game Theory for channel allocation in cognitive radio wireless sensor networks have been explored. The dependency of network metrics while designing utility functions especially in terms of reduction of interference levels has been detailed. Six different utility functions deduced by considering the following parameters under different network scenarios have been explored and each of the utility functions has been examined for their performance in terms of their level of interference. Case I: Using only SINR, Case II: Using I_to and I_from, Case III: Using I_to, I_fromand remaining energy, Case IV: Using I_to, I_from, remaining energy and cost, Case V: Using I_to, I_from, remaining energy, cost, and PU's detection, Case VI: Using Throughput. It is found that Case IV and V outperform other utility functions. The worst performing utility function is Case II while Case I and Case VI have similar performance. Considering the above cases, it shows that derivation of utility function while designing game-based channel allocation for CRWSN plays a major role in the general performance of the algorithm. Besides, it is vital to determine the correct network metrics while deriving the utility function. Hence, the design of utility function is of utmost importance. In the future, the work can be extended further by considering PU to be active, and further, the problem of joint channel allocation cum routing may be tackled.

\section{REFERENCES}

[1] J. Mitola and G. Q. Maguire, "Cognitive radio: making software radios more personal," IEEE Pers. Commun., vol. 6, no. 4, pp. 13-18, 1999, doi: 10.1109/98.788210.

[2] S. Haykin, "Cognitive radio: brain-empowered wireless communications," IEEE J. Sel. Areas Commun., vol. 23, no. 2, pp. 201-220, 2005, doi: 10.1109/JSAC.2004.839380.

[3] O. B. Akan, O. B. Karli, and O. Ergul, "Cognitive radio sensor networks," IEEE Netw., vol. 23, no. 4, pp. 34-40, 2009, doi: 10.1109/MNET.2009.5191144.

[4] J. Abolarinwa and A. Achonu, "Cognitive radio-based wireless sensor networks as next generation sensor network: Concept, problems and prospects 1," 2013.

[5] M. A. Matin, Spectrum Access and Management for Cognitive Radio Networks. Springer, 2017.

[6] G. P. Joshi, S. Y. Nam, and S. W. Kim, "Cognitive radio wireless sensor networks: applications, challenges and research trends," Sensors, vol. 13, no. 9, pp. 11196-11228, 2013, doi: 10.3390/s130911196.

[7] H.-Y. Shi, W.-L. Wang, N.-M. Kwok, and S.-Y. Chen, "Game theory for wireless sensor networks: a survey," Sensors, vol. 12, no. 7, pp. 9055-9097, 2012, doi: 10.3390/s120709055.

[8] A. Bhattarai, P. Komolkiti, and C. Aswakul, "Improved bandwidth allocation in Cognitive Radio Networks based on game theory," in 2013 10th International Conference on Electrical Engineering/Electronics, Computer, Telecommunications and Information Technology, pp. 1-6, 2013, doi:10.1109/ecticon.2013.6559481.

[9] A. Bhattarai, C. Charoenlarpnopparut, P. Suksompong, and P. Komolkiti, "Developing policies for channel allocation in Cognitive Radio Networks using Game Theory," in 2014 11th International Conference on Electrical Engineering/Electronics, Computer, Telecommunications and Information Technology (ECTI-CON), pp. 1-6, 2014, doi: 10.1109/ECTICon.2014.6839829.

[10] A. Bhattarai, C. Charoenlarpnopparut, P. Suksompong, and P. Komolkiti, "Policies for channel allocation in cognitive radio networks using game theory," ECTI Trans. Electr. Eng. Electron. Commun., vol. 14, no. 1, pp. 3648, 2016, doi: 10.1109/ECTICon.2014.6839829.

[11] H. Xu, H. Gao, C. Zhou, R. Duan, and X. Zhou, "Resource allocation in cognitive radio wireless sensor networks with energy harvesting,” Sensors, vol. 19, no. 23, p. 5115, 2019, doi: 10.3390/s19235115.

[12] P. Rai, M. Ghose, and H. K. D. Sarma, "A game theory-based framework for reliable and energy-efficient data delivery in cognitive radio wireless sensor network," Trends Commun. Cloud Big Data, pp. 31-39, 2020, doi: 10.1007/978-981-15-1624-5.

[13] S. R. Chatterjee, S. Ghosh, and M. Chakraborty, "Nash Bargaining in Resource Allocation for Cognitive Radio: A Review," Wirel. Pers. Commun., pp. 1-15, 2021.

[14] Q. Minhas, H. Mahmood, and H. Malik, "Channel selection for simultaneous move game in cognitive radio ad hoc networks," Wirel. Netw., vol. 22, no. 1, pp. 61-68, 2016, doi: 10.1007/s11276-015-0952-x.

[15] Q.-A. Minhas, M. A. Tawhid, and H. Mahmood, "Efficient power and channel allocation strategies in cooperative potential games for cognitive radio sensor networks," Int. J. Distrib. Sens. Netw., vol. 10, no. 6, p. 130130, 2014, doi: 10.1155/2014/130130.

[16] X.-C. Hao, X.-Y. Ru, X.-D. Li, and M.-J. Xin, "Energy efficient based channel assignment game algorithm for wireless sensor network," Wirel. Pers. Commun., vol. 85, no. 4, pp. 2749-2771, 2015.

[17] E. Romero, J. Blesa, A. Araujo, and O. Nieto-Taladriz, "A game theory based strategy for reducing energy consumption in cognitive WSN," Int. J. Distrib. Sens. Netw., vol. 10, no. 1, p. 965495, 2014, doi: $10.1155 / 2014 / 965495$.

[18] S. Garg, P. Mittal, and C. K. Nagpal, "A Novel Transmission Power Efficient Routing in Cognitive Radio Networks Using Game Theory," in Silicon Photonics \& High Performance Computing, Springer, pp. 35-44, 2018, doi: 10.1007/978-981-10-7656-5_5. 
[19] H. Idoudi, "Scheduling in Cognitive Radio Sensor Networks-An Auction-based Mechanism," in 2018 IFIP/IEEE International Conference on Performance Evaluation and Modeling in Wired and Wireless Networks (PEMWN), pp. 1-6, 2018, doi: 10.23919/PEMWN.2018.8548906.

[20] Z. Hao, J. Hou, J. Dang, X. Dang, and N. Qu, "Game algorithm based on link quality: Wireless sensor network routing game algorithm based on link quality," Int. J. Distrib. Sens. Netw., vol. 17, no. 2, p. 1550147721996248 , 2021, doi: $10.1177 / 1550147721996248$.

[21] P. Rai, M. K. Ghose, and H. K. D. Sarma, "An Analysis on the Impact of Utility Functions on the Performance of Game Theory Based Channel Allocation in Cognitive Radio Wireless Sensor Network," in 2020 IEEE International Conference on Advanced Networks and Telecommunications Systems (ANTS), pp. 1-6, 2020, doi: 10.1109/ANTS50601.2020.9342781.

[22] S. Lim, "Game-Theoretic Channel Allocation in Cognitive Radio Networks," Int. J. Electr. Comput. Eng., vol. 7 , no. 2, p. 986, 2017, doi: 10.11591/ijece.v7i2.pp986-991.

[23] S. Amiri-Doomari, G. Mirjalily, and J. Abouei, "Interference Aware Routing Game for Cognitive Radio Ad-hoc Networks," J. Telecommun. Inf. Technol., vol. 3, no. 3, pp. 55-63, 2018, doi: 10.26636/jtit.2018.110817.

[24] M. J. Osborne and others, An introduction to game theory, vol. 3. Oxford University press New York, 2004.

[25] Q. Zhu, Z. Yuan, J. B. Song, Z. Han, and T. Basar, "Interference aware routing game for cognitive radio multi-hop networks," IEEE J. Sel. Areas Commun., vol. 30, no. 10, pp. 2006-2015, 2012, doi: 10.1109/JSAC.2012.121115.

[26] J. Walrand and P. P. Varaiya, High-performance communication networks. Morgan Kaufmann, 2000.

\section{BIOGRAPHIES OF AUTHORS}

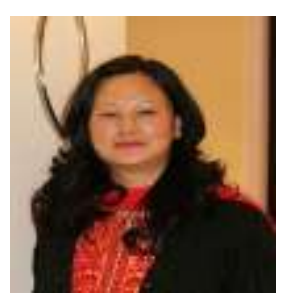

Prativa Rai is currently pursuing her Ph.D. from Sikkim Manipal University, Sikkim, India. She is also working as Associate Professor in Computer Science \& Engineering Department, Sikkim Manipal Institute of Technology, SMU, Sikkim, India. Her current research interests are in the area of Wireless Sensor Networks, Cognitive Radio Wireless Sensor Networks, Game Theory, Blockchain.

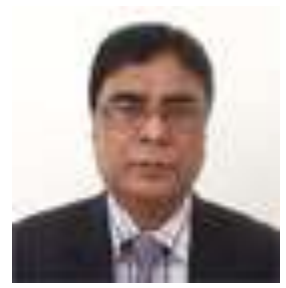

Prof. Mrinal Kanti Ghose presently working as a Distinguished Professor in Computer Engineering \& Applications, GLA University, Mathura-281406 (UP), India has coveted experience in research and academics for the last 37 years. He was associated with the Indian Space Research Organization as a Senior Scientist, followed by Dean (Academics) \& Professor in Sikkim Manipal Institute of Technology, Sikkim, India. Produced 22 Ph.Ds. in Computer engineering and allied fields, published more than 300 research papers in peer-reviewed international journals, chaired and organized many conferences, and act as a reviewer of many reputed journals. Research interests are Geo-Informatics, DIP, Soft computing, Data Mining, Network \& Information Security, Software Engineering, R \& QA, and Applied Statistics.

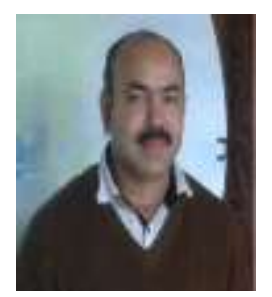

Dr. Hiren Kumar Deva Sarma is currently a Professor in the Department of Information Technology, Sikkim Manipal Institute of Technology, Sikkim. He has co-authored two books, edited three books, published more than eighty research papers in different International Journals, referred to International and National Conferences of repute. He is the recipient of the Young Scientist Award from the International Union of Radio Science (URSI) in the XVIII General Assembly 2005, held in New Delhi, India, and has received IEEE Early Adopter Award in 2014. His current research interests are wireless sensor networks, IoT, mobility management in IPv6-based networks, cognitive radio networks, information-centric networks, network security, robotics, distributed computing, and big data analytics. 